\title{
Workers' perceptions of climate change related extreme heat exposure in South Australia: a cross-sectional survey
}

\author{
Jianjun Xiang ${ }^{1,2}$, Alana Hansen $^{1}$, Dino Pisaniello ${ }^{1}$ and Peng Bi ${ }^{1 *}$
}

\begin{abstract}
Background: Occupational exposure to extreme heat without sufficient protection may not only increase the risk of heat-related illnesses and injuries but also compromise economic productivity. With predictions of more frequent and intense bouts of hot weather, workplace heat exposure is presenting a growing challenge to workers' health and safety. This study aims to investigate workers' perceptions and behavioural responses towards extreme heat exposure in a warming climate.
\end{abstract}

Methods: A cross-sectional questionnaire survey was conducted in 2012 in South Australia among selected outdoor industries. Workers' heat risk perceptions were measured in the following five aspects: concerns about heat exposure, attitudes towards more training, policy and guideline support, the adjustment of work habits, and degree of satisfaction of current preventive measures. Bivariate and multivariate logistic regression analyses were used to identify factors significantly associated with workers' heat perceptions.

Results: A total of 749 respondents participated in this survey, with a response rate of $50.9 \%$. A little more than half (51.2\%) of respondents were moderately or very much concerned about workplace heat exposure. Factors associated with workers' heat concerns included age, undertaking very physically demanding work, and the use of personal protective equipment, heat illness history, and injury experience during hot weather. Less than half (43.4 \%) of the respondents had received heat-related training. Workers aged 25-54 years and those with previous heat-related illness/injury history showed more supportive attitudes towards heat-related training. The provision of cool drinking water was the most common heat prevention measure. A little more than half (51.4\%) of respondents were satisfied with the current heat prevention measures. About two-thirds (63.8\%) of respondents agreed that there should be more heat-related regulations and guidelines for working during very hot weather. More than two-thirds $(68.8 \%)$ of the respondents were willing to adjust their current work habits to adapt to the likely increasing extreme heat, especially those with previous heat illness experience.

Conclusions: The findings suggest a need to strengthen workers' heat risk awareness and refine current heat prevention strategies in a warming climate. Further heat educational programmes and training should focus on those undertaking physically demanding work outdoors, in particular young workers and those over 55 years with low education levels.

Keywords: Perceptions, Heat stress, Workplace heat exposure, Climate change, Work-related injuries

\footnotetext{
* Correspondence: peng.bi@adelaide.edu.au

'School of Public Health, The University of Adelaide, Adelaide 5005, SA,

Australia

Full list of author information is available at the end of the article
} 


\section{Background}

Occupational exposure to extreme heat without sufficient protection may not only increase the risk of heatrelated illnesses and injuries [1-5], but also compromise economic productivity by reducing work efficiency [6] and the time that work can safely be undertaken outdoors during the hottest part of the day [7]. Workplace heat gain can be a combination of heat from the external thermal environment and internal heat generation by metabolism associated with physical activity [8]. External heat exposure sources in the workplace include weatherrelated and process-generated heat. With predictions of more frequent and intense bouts of hot weather [9], workplace heat exposure is presenting a growing challenge to workers' health and safety [10-12], especially for outdoor workers and those undertaking physically demanding work in hot environments [13].

Evidence shows that Australia would be at moderate to high risk of occupational heat strain for outdoor workers if temperatures increase by $3{ }^{\circ} \mathrm{C}$ [14]. Dangerous days (days when there is a $2.5{ }^{\circ} \mathrm{C}$ increase in body temperature in less than 2 hours) for Australian outdoor workers may increase to 15-27 days per year by 2070 compared with 1 day per year at present [15]. Several heat-related deaths have recently been reported in Australian workplaces [16-18], raising increased concerns over workplace heat exposure. The potential heat-related impact of climate change on workplaces may be even worse in South Australia (SA) than the Australian national average level, as the average maximum temperature has increased at a faster rate than the national average since 1950 [19]. Adelaide, the capital of SA, has a Mediterranean climate, characterised with very limited rainfall during hot dry summers and maximum temperatures reaching as high as $46.1{ }^{\circ} \mathrm{C}$. According to weather projections for Adelaide, the average number of days with temperatures over $35^{\circ} \mathrm{C}$ will triple by 2070 [19]. Some studies in Adelaide have found that heat stress levels were high in some occupations (e.g., miners, shearers, and road maintenance workers) [13], and work-related injury claims were significantly associated with ambient temperatures and heatwaves $[2,3]$.

Heat-related illnesses and injuries are largely preventable. Current heat prevention strategies include engineering controls, administrative control, personal protection, education and training, and regulations [8]. Effective heat stress management needs comprehensive efforts, cooperation, and support from a wide variety of stakeholders such as employees, employers, and occupational health professionals. An investigation of how people perceive the risk of workplace heat exposure may be helpful to identify potential heat prevention and adaptation barriers, to make the allocation of heat prevention resources more focused, and to refine current heat policies to make them more practicable and operational.

To raise awareness of the harm caused by weatherrelated extreme heat exposure, the US Occupational Safety and Health Administration lunched the nationalwide Heat Illness Prevention Campaign in 2011 [20]. Prior to the possible development of heat stress management, there is a need to investigate the baseline information regarding how people perceive the risk of heat stress. To the authors' knowledge, only two qualitative studies have preliminarily explored Australian workers' heat risk perceptions $[11,21]$, and suggested that there was a misalignment of perceived occupational health risk, given Australia's existing thermal environments and predicted increasingly hot summers [9]. The present quantitative study using a questionnaire survey is the first in Australia with aims to investigate workers' perceptions and behavioural responses regarding extreme heat exposure, and identify factors affecting individuals' heat perceptions.

\section{Methods}

A cross-sectional questionnaire survey was conducted between 15 August and 6 November 2012 in Adelaide, South Australia, amongst selected workers and trades apprentices at high risk of workplace heat exposure. The average daily maximum temperature was $20.6{ }^{\circ} \mathrm{C}$ in Adelaide during the study period, ranging from 12.4 to $35.0{ }^{\circ} \mathrm{C}$.

\section{Questionnaire design}

The questionnaire was developed after a comprehensive review of the literature on heat exposure and occupational health [13]. The draft questionnaire was reviewed by relevant experts and piloted among 10 outdoor workers in Adelaide. Relevant revisions were made to ensure all questions were clear and understandable.

The final questionnaire (Additional file 1) consisted of 5 demographic variables and 20 questions involving aspects of the working environments, previous history of heat-related illness and injury, heat prevention management, and perceptions of workplace extreme heat exposure. Some questions had both closed and open-ended responses. Some multiple choice questions investigated individual work habits, access to heat stress prevention information, and heat prevention measures. Likert-scale questions were used to measure heat-related perceptions and attitudes.

\section{Participant recruitment}

The inclusion criteria for participants were those working outdoors, or indoors without air conditioning, in the following four industries: "agriculture, fishing and forestry", "construction", "electricity, gas and water" (the 
latter refers to workers engaged in the provision of public utilities), and "mining", according to the findings of previous studies [2, 3] and relevant literature [13].

With the support of SafeWork South Australia (SWSA), the local occupational health and safety $(\mathrm{OH} \& \mathrm{~S})$ regulator, a total of 164 employers who reported relatively more injury claims during the period of 2001-2010 in SA were invited to participate in the study. These employers provided assistance in the distribution of survey questionnaires, envelopes, and information sheets (Additional file 2 ) to their workers who met the inclusion criteria.

Young workers appear to be relatively at higher risk of heat-related injuries in the workplace [22], however they may be underrepresented due to the aging workforce. Moreover, young workers were usually less inclined to participate in surveys due to various reasons. Therefore, apprentices at local TAFE (Technical and Further Education) colleges were also recruited to represent young workers, a large proportion of whom undertook their studies part time while working as apprentices. In total, 840 TAFE trainees and apprentices enrolled in the courses likely to lead to outdoor work in the "agriculture, forestry and fishing", "construction", "electricity, gas and water", "manufacturing", and "mining" industries were invited to participate in the survey. After initial contact and meeting with the TAFE College management team and relevant lecturers, questionnaires were distributed by the course lecturers.

Participants filled out the questionnaires independently in their own time. Completed questionnaires were returned by participants using supplied reply-paid envelopes. All participants were therefore free of any potential pressure from their employers and supervisors during the completion of the questionnaire. Oral consent was obtained from each participant. The study was approved by the Human Research Ethics Committee at the University of Adelaide (H-200-2011).

\section{Data analyses}

Data entry and validation were performed using 'Microsoft Excel 2007', and imported into Stata statistical software (version 12.0, College Station, Texas, USA) for data manipulation and analyses. The "SVY" commands of Stata were used to calculate odds ratios (OR) and $95 \%$ confidence intervals for the prevalence estimates [23].

Five indicators were used to represent workers' perceptions of workplace heat exposure from different aspects. They were (1) workers' concerns about heat exposure, (2) attitudes towards more training, (3) policy and guideline support, (4) the adjustment of work habits, and (5) degree of satisfaction of current preventive measures. To identify the factors significantly associated with perceptions of workplace heat exposure, bivariate and multivariate logistic regression analyses were conducted using a stepwise backwards model. All variables with statistical significance of $p<0.05$ were included in the final model.

\section{Results \\ Demographic characteristics}

A total of 1,471 questionnaires were distributed and 749 were returned, with a response rate of $50.9 \%$. The 749 respondents consisted of 511 (68.2 \%) TAFE apprentices and 238 (31.8\%) established workers. Among the 511 TAFE apprentices, 91.4 \% were part-time, with $8.6 \%$ full-time TAFE students. As shown in Table 1, the majority $(96.0 \%)$ of respondents were male, and young people ( $\leq 24$ years) accounted for more than half $(53.5 \%)$ of all respondents. In terms of highest educational attainment, more than half (51.3\%) of the respondents had completed high school, $42.6 \%$ had a trade certificate, and $6.1 \%$ had a university degree. Almost three-quarters $(73.2 \%)$ of respondents were employed as tradespersons and related workers. Labourers accounted for $5.7 \%$ of respondents. More than half $(51.0 \%)$ of respondents mainly undertook work outdoors. The construction industry had the highest percentage $(37.1 \%)$ of respondents, followed by manufacturing $(27.5 \%)$, mining (14.6\%), 'electricity, gas and water' (13.1\%), and 'agriculture, forestry \& fishing' (2.0\%). Approximately three-quarters $(75.9 \%)$ of respondents considered that their jobs were moderately or highly physically demanding, while $38.1 \%$ reported that they worked close to heat sources. Two-thirds $(67.8 \%)$ of respondents were required to wear personal protective equipment (PPE), which may include overalls, gloves, helmets, goggles, respirators, face masks, and high visibility clothing etc.

\section{Heat-related illnesses and injury experience during very hot weather}

Overall, 279 (37.2 \%) of respondents had experienced heat illnesses during hot days. The most common type of heat illnesses reported were heat exhaustion (60.6\%), followed by heat rashes (43.0 \%), heat stroke (26.2 \%), and heat cramps (18.3\%) (Data not shown, Question 12, Additional file 1). About one-quarter (25.9 \%) of respondents experienced heat-related injuries during very hot weather. More than half (54.1\%) of the injuries were caused by burns, $44.3 \%$ by falls, trips and slips, $27.8 \%$ by hitting objects, and $10.3 \%$ by being hit by moving objects (Question 15, Additional file 1).

When asked if they had witnessed an injury to another person during hot weather (Question 16, Additional file 1), $25.2 \%$ of participants responded that they had. The most common type of injuries witnessed during very hot weather was falls, trips and slips $(55.0 \%)$, followed by burns (42.3\%), hitting objects (22.8\%), and being hit by moving objects (17.5\%). 
Table 1 Perceptions of workplace heat exposure: prevalence estimates and $95 \% \mathrm{Cl}$ by different subgroups

\begin{tabular}{|c|c|c|c|c|c|c|c|}
\hline Independent variable & $n$ & $\%$ & $\begin{array}{l}\text { Concern for } \\
\text { extreme heat } \\
\% \text { ( } 95 \% \text { Cl) }\end{array}$ & $\begin{array}{l}\text { Positive attitude } \\
\text { for more training } \\
\%(95 \% \text { Cl) }\end{array}$ & $\begin{array}{l}\text { Positive attitude } \\
\text { for more regulation } \\
\% \text { ( } 95 \% \text { Cl) }\end{array}$ & $\begin{array}{l}\text { Positive attitude for } \\
\text { adjusting work habits } \\
\%(95 \% \text { Cl) }\end{array}$ & $\begin{array}{l}\text { Satisfaction degree for } \\
\text { preventive measures } \\
\%(95 \% \text { Cl) }\end{array}$ \\
\hline Total & 749 & 100 & $51.2(50.0-57.4)$ & $56.3(50.1-61.9)$ & $63.8(57.3-69.9)$ & $68.8(63.4-73.7)$ & $51.4(42.6-60.0)$ \\
\hline \multicolumn{8}{|l|}{ Gender } \\
\hline Male & 719 & 96.0 & $51.1(44.7-57.5)$ & $56.5(51.1-61.7)$ & $64.1(57.5-70.2)$ & $69.2(63.9-74.0)$ & $50.9(42.3-59.5)$ \\
\hline Female & 30 & 4.0 & $53.3(34.1-71.6)$ & $53.3(28.9-76.3)$ & $56.7(37.7-78.9)$ & $60.0(40.5-76.8)$ & $62.1(39.8-80.2)$ \\
\hline \multicolumn{8}{|l|}{ Age group } \\
\hline$\leq 24$ & 401 & 53.5 & $44.0(38.5-49.6)$ & $50.0(43.1-56.9)$ & $66.9(58.7-74.3)$ & $66.4(59.2-73.0)$ & $42.0(35.6-48.7)$ \\
\hline $25-34$ & 101 & 13.5 & $58.5(48.9-67.5)$ & $63.5(56.4-70.1)$ & $74.0(63.8-82.0)$ & $76.3(64.0-85.5)$ & $44.1(31.7-57.3)$ \\
\hline $35-54$ & 161 & 21.5 & $63.5(48.3-76.4)$ & $66.7(53.2-77.9)$ & $59.0(48.2-69.0)$ & $73.7(63.2-82.1)$ & $68.8(58.1-77.9)$ \\
\hline$\geqq 55$ & 86 & 11.5 & $59.8(44.3-73.5)$ & $64.6(46.5-79.4)$ & $48.8(37.2-60.5)$ & $65.9(51.3-78.0)$ & $70.9(55.7-82.5)$ \\
\hline \multicolumn{8}{|l|}{ Education level } \\
\hline High school & 384 & 51.3 & $46.8(40.7-53.0)$ & $53.4(46.6-60.1)$ & $66.2(58.4-73.2)$ & $67.6(60.5-73.9)$ & $46.4(37.1-55.9)$ \\
\hline Trade certificate & 319 & 42.6 & $56.1(48.5-63.3)$ & $58.7(52.6-64.5)$ & $64.0(56.7-70.7)$ & $69.8(63.5-75.4)$ & $51.8(42.0-61.5)$ \\
\hline University degree & 46 & 6.1 & $53.3(43.4-63.0)$ & $62.2(49.3-73.6)$ & $42.2(24.1-62.7)$ & $73.3(52.8-87.1)$ & 86.7 (71.9-94.3) \\
\hline \multicolumn{8}{|l|}{ Occupation } \\
\hline $\begin{array}{l}\text { Tradespersons and } \\
\text { related workers }\end{array}$ & 548 & 73.2 & $50.5(44.5-56.4)$ & $55.1(49.5-60.6)$ & $66.7(59.9-72.8)$ & $70.0(65.3-74.4)$ & $46.6(38.5-55.0)$ \\
\hline $\begin{array}{l}\text { Clerical and administrative } \\
\text { workers }\end{array}$ & 30 & 4.0 & $40.0(19.8-64.2)$ & $70.0(28.1-93.3)$ & $46.7(21.6-73.6)$ & $66.7(36.6-87.4)$ & $86.2(64.8-95.5)$ \\
\hline $\begin{array}{l}\text { Machinery operators and } \\
\text { drivers }\end{array}$ & 55 & 7.3 & $65.5(32.1-88.4)$ & $58.2(27.8-83.4)$ & $61.8(36.9-81.7)$ & $54.6(42.9-65.7)$ & $63.6(42.7-80.4)$ \\
\hline $\begin{array}{l}\text { Labourers and related } \\
\text { workers }\end{array}$ & 43 & 5.7 & $51.2(32.5-69.5)$ & $55.8(47.7-63.7)$ & $62.8(38.3-82.1)$ & $69.8(54.4-81.7)$ & $45.2(30.0-61.4)$ \\
\hline Full-time TAFE students & 44 & 5.9 & $40.9(21.0-79.0)$ & $54.6(39.6-68.7)$ & $59.1(48.3-69.1)$ & $67.4(53.4-78.9)$ & $56.1(35.6-74.7)$ \\
\hline Professionals & 16 & 2.1 & $81.3(62.0-92.0)$ & $68.8(30.0-91.9)$ & $37.5(15.7-65.9)$ & $81.3(53.1-94.3)$ & $87.5(51.5-97.9)$ \\
\hline Other & 13 & 1.7 & $46.2(17.2-77.9)$ & $61.5(24.9-88.5)$ & $46.2(16.7-78.6)$ & $69.2(37.7-89.3)$ & 76.9 (34.1-95.6) \\
\hline \multicolumn{8}{|l|}{ Industry } \\
\hline $\begin{array}{l}\text { Agriculture, forestry \& } \\
\text { fishing }\end{array}$ & 15 & 2.0 & $50.0(24.4-75.6)$ & $62.5(41.3-79.8)$ & $66.7(49.4-80.4)$ & $58.3(37.2-76.8)$ & $69.6(51.6-83.0)$ \\
\hline Mining & 109 & 14.6 & $57.0(45.5-67.8)$ & $51.3(35.2-67.2)$ & $59.1(38.4-77.0)$ & $67.3(44.9-83.8)$ & $59.5(32.6-81.7)$ \\
\hline Manufacturing & 206 & 27.5 & $44.2(37.9-50.8)$ & $49.5(32.5-66.7)$ & $67.5(45.7-83.6)$ & $66.7(48.7-80.8)$ & $36.4(25.8-48.6)$ \\
\hline Construction & 278 & 37.1 & $52.4(38.8-65.6)$ & $58.8(51.6-65.6)$ & $67.0(59.5-73.8)$ & $69.3(63.9-74.3)$ & $50.2(43.2-57.2)$ \\
\hline Electricity, gas \& water & 98 & 13.1 & $57.6(33.3-78.7)$ & $67.9(60.7-74.4)$ & $52.8(43.4-62.1)$ & $75.5(64.4-84.0)$ & $71.7(43.8-89.2)$ \\
\hline Other & 43 & 5.7 & $38.5(9.4-78.9)$ & $53.9(28.8-77.1)$ & $61.5(23.8-89.1)$ & $69.2(35.6-90.1)$ & $46.2(12.8-83.3)$ \\
\hline \multicolumn{8}{|l|}{ Workplace environment } \\
\hline Completely indoors & 59 & 7.9 & $45.7(37.0-54.8)$ & $74.6(57.5-86.4)$ & $71.2(53.9-83.9)$ & $69.0(52.1-82.0)$ & $37.3(21.4-56.5)$ \\
\hline Mainly indoors & 282 & 37.7 & $44.4(35.7-53.6)$ & $52.5(43.0-61.8)$ & $58.5(48.4-68.0)$ & $68.6(62.7-74.0)$ & $59.5(44.6-72.8)$ \\
\hline Completely outdoors & 82 & 10.9 & $71.6(55.7-83.5)$ & $63.4(49.5-75.4)$ & $70.7(58.6-80.5)$ & $63.8(46.3-78.2)$ & $44.3(29.9-59.7)$ \\
\hline Mainly outdoors & 300 & 40.1 & $53.2(48.6-58.3)$ & $55.3(50.0-60.6)$ & $63.7(57.1-69.7)$ & $68.9(61.6-75.4)$ & $49.0(41.5-56.5)$ \\
\hline \multicolumn{8}{|l|}{ Physically demanding } \\
\hline Not at all & 49 & 6.5 & $40.4(29.3-52.7)$ & $63.8(38.8-83.1)$ & $51.1(32.4-69.4)$ & $73.9(61.8-83.2)$ & $82.6(69.0-91.0)$ \\
\hline A little & 132 & 17.6 & $44.5(35.9-53.5)$ & $53.1(39.1-66.6)$ & $47.7(35.5-60.2)$ & $59.8(47.3-71.2)$ & $70.6(53.5-83.4)$ \\
\hline Moderately & 280 & 37.4 & $48.0(40.3-55.8)$ & $56.1(50.3-61.8)$ & $64.0(56.6-70.8)$ & $72.0(65.4-77.7)$ & $50.9(44.8-57.0)$ \\
\hline Very much & 288 & 38.5 & $59.9(50.7-68.4)$ & $56.6(50.0-63.1)$ & $72.7(66.3-78.4)$ & $69.5(63.9-74.6)$ & $37.8(30.0-46.5)$ \\
\hline
\end{tabular}


Table 1 Perceptions of workplace heat exposure: prevalence estimates and $95 \% \mathrm{Cl}$ by different subgroups (Continued)

\begin{tabular}{|c|c|c|c|c|c|c|c|}
\hline \multicolumn{8}{|c|}{ Work close to heat sources } \\
\hline Yes & 285 & 38.1 & $52.4(46.7-58.0)$ & $56.8(49.9-63.4)$ & $67.0(59.1-74.0)$ & $69.8(64.9-74.2)$ & $44.8(35.6-54.4)$ \\
\hline No & 464 & 61.9 & $49.3(39.8-58.8)$ & $56.5(46.5-66.0)$ & $59.3(50.8-67.3)$ & $68.4(60.1-75.6)$ & $61.7(50.5-71.7)$ \\
\hline \multicolumn{8}{|c|}{$\begin{array}{l}\text { Use of personal protective } \\
\text { equipment }\end{array}$} \\
\hline Yes & 508 & 67.8 & $55.8(49.7-61.8)$ & $58.0(52.4-63.3)$ & $64.2(58.0-70.0)$ & $69.9(64.4-74.8)$ & $50.1(40.5-59.7)$ \\
\hline No & 241 & 32.2 & $41.4(34.0-49.4)$ & $52.9(45.0-60.7)$ & $62.9(49.7-74.5)$ & $66.5(58.1-74.0)$ & $54.1(40.7-66.9)$ \\
\hline \multicolumn{8}{|c|}{ Heat illness experience } \\
\hline Yes & 279 & 37.2 & $62.4(52.2-71.5)$ & $65.2(56.8-72.8)$ & $70.3(62.9-76.7)$ & $73.9(67.8-79.2)$ & $38.5(30.6-47.0)$ \\
\hline No & 470 & 62.8 & $44.5(39.5-49.6)$ & $51.4(44.2-58.5)$ & $60.4(52.9-67.4)$ & $66.1(60.1-71.6)$ & $59.0(48.2-69.1)$ \\
\hline \multicolumn{8}{|c|}{ Heat-related injury experience } \\
\hline Yes & 194 & 25.9 & $67.0(57.0-75.7)$ & $65.0(57.0-72.1)$ & $72.2(60.6-81.4)$ & $71.2(62.7-78.4)$ & $29.5(23.5-36.2)$ \\
\hline No & 555 & 74.1 & $45.6(39.9-51.5)$ & $53.3(47.5-59.1)$ & $60.9(54.0-67.4)$ & $68.0(62.5-73.0)$ & $59.0(48.8-68.5)$ \\
\hline
\end{tabular}

\section{Risk perceptions of workplace heat exposure}

Overall, $51.2 \%$ of respondents were moderately or very much concerned about the risk of heat illness at work during very hot weather (Table 1 ). Results of stepwise logistic regression analyses indicated that workplace heat concern levels increased with the increasing age. As shown in Table 2, workers in the three age groups (25$34,35-54$, and $\geq 55$ years) were respectively 1.82 (95\% CI 1.18-2.80), 2.73 (95\%CI 1.45-5.12), and 2.77 (95 \% CI 1.73-4.43) times more concerned about heat stress than those aged $\leq 24$ years. Other factors significantly associated with workers' heat stress concerns include undertaking heavily physically demanding work $(\mathrm{OR}=2.77$, $95 \%$ CI 1.73-4.43), wearing PPE $(\mathrm{OR}=1.47$, $95 \% \mathrm{CI}$ 1.13-1.91), having a history of heat illness $(\mathrm{OR}=1.57$, $95 \%$ CI 1.01-2.45) and having experienced an injury during hot weather $(\mathrm{OR}=2.05,95 \% \mathrm{CI} 1.47-2.86)$.

About $56.3 \%$ of respondents replied that there was a need for more heat-related training for workers to reduce the risk of heat stress (Table 1). As shown in Table 2, workers aged 25-54 years showed more supportive attitudes towards heat-related trainings than younger ( $\leq 24$ years) and older workers ( $\geq 55$ years). In addition, workers with previous history of heat illness $(\mathrm{OR}=1.58,95 \% 1.07-2.58)$ and injury during hot weather $(\mathrm{OR}=1.44,95 \% 1.09-1.91)$ were more interested in heat-related trainings.

Results showed that $63.8 \%$ of respondents agreed that there should be more heat-related regulations and guidelines for working during very hot weather (Table 1). Regarding the reasons why the remaining $36.2 \%$ held the opposite view, answers from a subsequent question (Question 20, Additional file 1) suggested that over half (52.3\%) thought "there are enough heat regulations", while $21.5 \%$ considered workplace heat exposure to not be a serious problem. Multiple logistic regression analyses suggested that very physically demanding work
$(\mathrm{OR}=2.38,95 \% \mathrm{CI}$ 1.21-4.66) and heat illness experience $(\mathrm{OR}=1.39,95 \%$ CI 1.05-1.83) were the two factors associated with workers' attitudes toward more heatrelated policy support (Table 2).

More than two-thirds (68.8 \%) of respondents answered they were willing to adjust their current work habits to adapt to the impact of extreme heat (Table 1). As to the reasons the remaining $31.2 \%$ did not consider the adjustment of work habits during hot weather (Question 24, Additional file 1), one-third (33.3 \%) thought "Enough has been done already", followed by "I don't think I am at risk" (30.3 \%), and "I don't think it is a serious problem" (24.4\%). Multiple logistic regression analyses indicated that workers with previous heat illness experience $(\mathrm{OR}=1.45$, 95 \% CI 1.15-1.84) showed stronger willingness to adjust their heat-related work habits.

More than half $(51.4 \%)$ of respondents were satisfied with the heat prevention measures currently adopted in South Australian workplaces (Table 1). Factors significantly associated with workers' satisfaction in terms of current heat prevention measures include: age (35-54 and $\geq 55$ years), education level (university degree), performing physically demanding work, previous heat illness experience and injury experience during hot weather (Table 2).

\section{Personal behaviours during hot days}

The majority $(64.4 \%)$ of respondents drank water regularly during work, while $16.4 \%$ responded that they only drank when feeling thirsty, and $15.2 \%$ answered that they drank plenty of fluids before starting work. About one-fifth of participants' claimed all of these drinking habits in the workplace were applicable (Question 10, Additional file 1).

In terms of the main sources of information about heat prevention, as shown in Fig. 1, heat-related training (49.7\%) and learning at the workplace (48.9\%) were the 
Table 2 Factors associated with attitude and perception for workplace heat exposure: bivariate analysis (unadjusted) and multiple stepwise logistic regressions (adjusted)

\begin{tabular}{|c|c|c|c|c|c|c|c|c|c|c|}
\hline \multirow{2}{*}{ Independent variable } & \multicolumn{2}{|c|}{ Concern for extreme heat } & \multicolumn{2}{|c|}{ Positive attitude for more training } & \multicolumn{2}{|c|}{$\begin{array}{l}\text { Positive attitude for more } \\
\text { regulations }\end{array}$} & \multicolumn{2}{|c|}{$\begin{array}{l}\text { Positive attitude for adjusting } \\
\text { work habits }\end{array}$} & \multicolumn{2}{|c|}{$\begin{array}{c}\text { Satisfaction degree for preventive } \\
\text { measures }\end{array}$} \\
\hline & $\begin{array}{c}\text { Unadjusted OR* } \\
(95 \% \mathrm{Cl})\end{array}$ & $\begin{array}{c}\text { Adjusted OR } \\
(95 \% \mathrm{Cl})\end{array}$ & $\begin{array}{l}\text { Unadjusted OR } \\
(95 \% \mathrm{Cl})\end{array}$ & $\begin{array}{l}\text { Adjusted OR } \\
(95 \% \mathrm{Cl})\end{array}$ & $\begin{array}{l}\text { Unadjusted OR } \\
(95 \% \mathrm{Cl})\end{array}$ & $\begin{array}{l}\text { Adjusted OR } \\
(95 \% \mathrm{Cl})\end{array}$ & $\begin{array}{l}\text { Unadjusted OR } \\
(95 \% \mathrm{Cl})\end{array}$ & $\begin{array}{c}\text { Adjusted OR } \\
(95 \% \mathrm{Cl})\end{array}$ & $\begin{array}{l}\text { Unadjusted OR } \\
(95 \% \mathrm{Cl})\end{array}$ & $\begin{array}{l}\text { Adjusted OR } \\
(95 \% \mathrm{Cl})\end{array}$ \\
\hline \multicolumn{11}{|l|}{ Gender } \\
\hline Female & 1.00 & & 1.00 & & 1.00 & & 1.00 & & 1.00 & \\
\hline Male & $0.92(0.44-1.91)$ & & $1.13(0.49-2.65)$ & & $1.37(0.66-2.82)$ & & $1.50(0.76-2.95)$ & & $0.63(0.29-1.39)$ & \\
\hline \multicolumn{11}{|l|}{ Age group } \\
\hline$\leq 24$ & 1.00 & 1.00 & 1.00 & 1.00 & 1.00 & & 1.00 & & 1.00 & 1.00 \\
\hline $25-34$ & $1.80(1.25-2.57)$ & $1.82(1.18-2.80)$ & $1.74(1.24-2.45)$ & $1.75(1.27-2.39)$ & $1.40(0.76-2.61)$ & & $1.63(0.77-3.48)$ & & $1.09(0.65-1.82)$ & $0.94(0.61-1.44)$ \\
\hline $35-54$ & $2.21(1.18-4.16)$ & $2.73(1.45-5.12)$ & $2.00(1.06-3.79)$ & $1.96(1.05-3.65)$ & $0.71(0.43-1.17)$ & & $1.42(0.83-2.42)$ & & $3.05(1.92-4.85)$ & $2.54(1.66-3.88)$ \\
\hline$\geq 55$ & $1.19(1.01-3.52)$ & $2.77(1.73-4.43)$ & $1.83(0.87-3.83)$ & $1.93(0.99-3.76)$ & $0.47(0.28-0.80)$ & & $0.98(0.51-1.85)$ & & $3.36(1.61-7.00)$ & $2.12(1.00-4.48)$ \\
\hline \multicolumn{11}{|l|}{ Education level } \\
\hline High school & 1.00 & & 1.00 & & 1.00 & & 1.00 & & 1.00 & 1.00 \\
\hline Trade certificate & $1.45(1.14-1.84)$ & & $1.24(0.92-1.66)$ & & $0.91(0.66-1.24)$ & & $1.11(0.77-1.61)$ & & $1.24(0.83-1.87)$ & $1.12(0.75-1.67)$ \\
\hline University degree & $1.30(0.85-1.99)$ & & $1.44(0.94-2.19)$ & & $0.37(0.17-0.83)$ & & $1.32(0.59-2.93)$ & & $7.51(2.73-20.69)$ & $4.79(1.65-13.94)$ \\
\hline \multicolumn{11}{|l|}{ Occupation } \\
\hline Clerical and administrative workers & 1.00 & & 1.00 & & 1.00 & & 1.00 & & 1.00 & \\
\hline Tradespersons and related workers & $1.53(0.72-3.24)$ & & $0.53(0.13-2.16)$ & & $2.28(0.90-5.76)$ & & $1.17(0.46-3.00)$ & & $0.14(0.05-0.41)$ & \\
\hline Machinery operators and drivers & $2.84(0.65-12.45)$ & & $0.60(0.20-1.74)$ & & $1.85(0.74-4.65)$ & & $0.60(0.26-1.40)$ & & $0.28(0.11-0.69)$ & \\
\hline Labourers and related workers & $1.57(0.65-3.79)$ & & $0.54(0.13-2.20)$ & & $1.93(0.41-9.05)$ & & $1.15(0.44-3.00)$ & & $0.13(0.04-0.39)$ & \\
\hline Full-time TAFE students & $1.04(0.36-2.99)$ & & $0.51(0.12-2.23)$ & & $0.98(0.21-4.62)$ & & $1.04(0.35-3.05)$ & & $0.20(0.07-0.63)$ & \\
\hline Professionals & $6.50(2.20-19.20)$ & & $0.94(0.17-5.16)$ & & $0.69(0.23-2.07)$ & & $2.17(0.75-6.27)$ & & $1.12(0.16-7.78)$ & \\
\hline Other & $1.29(0.51-3.21)$ & & $0.69(0.21-2.20)$ & & $0.98(0.21-4.62)$ & & $1.13(0.42-2.99)$ & & $0.53(0.07-3.85)$ & \\
\hline \multicolumn{11}{|l|}{ Industry } \\
\hline Manufacturing & 1.00 & & 1.00 & & 1.00 & & 1.00 & & 1.00 & \\
\hline Agriculture, forestry \& fishing & $1.26(0.65-2.46)$ & & $1.70(0.81-3.56)$ & & $0.97(0.43-2.19)$ & & $0.70(0.33-1.49)$ & & $3.99(2.22-7.18)$ & \\
\hline Mining & $1.67(1.10-2.55)$ & & $1.07(0.50-2.31)$ & & $0.70(0.27-1.83)$ & & $1.03(0.41-2.60)$ & & $2.56(0.99-6.60)$ & \\
\hline Construction & $1.39(0.81-2.36)$ & & $1.45(0.77-2.74)$ & & $0.98(0.45-2.12)$ & & $1.13(0.60-2.14)$ & & $1.76(1.12-2.77)$ & \\
\hline Electricity, gas \& water & $1.71(0.94-3.11)$ & & $2.16(1.20-3.89)$ & & $0.77(0.13-4.44)$ & & $1.54(0.80-2.97)$ & & $4.42(2.05-9.56)$ & \\
\hline Other & $0.79(0.18-3.38)$ & & $1.19(0.42-3.35)$ & & $0.54(0.26-1.13)$ & & $1.13(0.30-4.19)$ & & $1.50(0.42-5.34)$ & \\
\hline \multicolumn{11}{|l|}{ Workplace environment } \\
\hline Completely indoors & 1.00 & & 1.00 & & 1.00 & & 1.00 & & 1.00 & \\
\hline Mainly indoors & $0.95(0.64-1.42)$ & & $0.38(0.19-0.74)$ & & $0.57(0.37-0.87)$ & & $0.98(0.54-1.80)$ & & $2.47(1.80-3.38)$ & \\
\hline Completely outdoors & $2.99(1.44-6.22)$ & & $0.59(0.24-1.46)$ & & $0.98(0.44-2.18)$ & & $0.79(0.33-1.91)$ & & $1.34(0.56-3.17)$ & \\
\hline Mainly outdoors & $1.19(0.70-2.01)$ & & $0.42(0.21-0.86)$ & & $0.71(0.37-1.38)$ & & $1.00(0.54-1.84)$ & & $1.61(0.77-3.39)$ & \\
\hline \multicolumn{11}{|l|}{ Physically demanding } \\
\hline Not at all & 1.00 & 1.00 & 1.00 & & 1.00 & 1.00 & 1.00 & & 1.00 & 1.00 \\
\hline A little & $1.18(0.81-1.73)$ & $1.26(0.87-1.83)$ & $0.64(0.32-1.28)$ & & $0.87(0.55-1.39)$ & $0.87(0.56-1.34)$ & $0.53(0.33-0.83)$ & & $0.51(0.22-1.19)$ & $0.67(0.28-1.58)$ \\
\hline Moderately & $1.36(0.77-2.40)$ & $1.73(0.92-3.28)$ & $0.72(0.30-1.77)$ & & $1.71(0.79-3.68)$ & $1.66(0.79-3.49)$ & $0.91(0.58-1.41)$ & & $0.21(0.11-0.44)$ & $0.43(0.24-0.79)$ \\
\hline Very much & $2.20(1.27-3.82)$ & $2.77(1.73-4.43)$ & $0.74(0.26-1.92)$ & & $2.56(1.28-5.11)$ & $2.38(1.21-4.66)$ & $0.80(0.54-1.19)$ & & $0.13(0.07-0.24)$ & $0.31(0.16-0.60)$ \\
\hline \multicolumn{11}{|l|}{ Work close to heat sources } \\
\hline No & 1.00 & & 1.00 & & 1.00 & & 1.00 & & 1.00 & \\
\hline Yes & $1.21(0.83-1.52)$ & & $1.02(0.63-1.67)$ & & $1.41(0.94-2.12)$ & & $1.08(0.80-1.46)$ & & $0.51(0.33-0.79)$ & \\
\hline \multicolumn{11}{|l|}{ Use of personal protective equipment } \\
\hline No & 1.00 & 1.00 & 1.00 & & 1.00 & & 1.00 & & 1.00 & \\
\hline Yes & $1.79(1.34-2.40)$ & $1.47(1.13-1.91)$ & $1.23(0.95-1.58)$ & & $1.06(0.63-1.77)$ & & $1.17(0.84-1.62)$ & & $0.85(0.50-1.44)$ & \\
\hline \multicolumn{11}{|l|}{ Heat illness experience } \\
\hline No & 1.00 & 1.00 & 1.00 & 1.00 & 1.00 & 1.00 & 1.00 & 1.00 & 1.00 & 1.00 \\
\hline Yes & $2.07(1.41-3.02)$ & $1.57(1.01-2.45)$ & $1.77(1.16-2.72)$ & $1.58(1.07-2.58)$ & $1.55(1.16-2.07)$ & $1.39(1.05-1.83)$ & $1.45(1.15-1.84)$ & $1.45(1.15-1.84)$ & $0.43(0.29-0.65)$ & $0.53(0.36-0.76)$ \\
\hline \multicolumn{11}{|c|}{ 政 } \\
\hline No & 1.00 & 1.00 & 1.00 & 1.00 & 1.00 & & 1.00 & & 1.00 & 1.00 \\
\hline Yes & $2.42(1.71-3.44)$ & $2.05(1.47-2.86)$ & $1.62(1.23-2.14)$ & $1.44(1.09-1.91)$ & $1.66(0.99-2.80)$ & & $1.17(0.84-1.63)$ & & $0.29(0.20-0.43)$ & $0.38(0.27-0.54)$ \\
\hline
\end{tabular}

most common way for respondents to obtain such information, followed by information from friends and families $(22.4 \%)$, colleagues (21.6\%), TV and radio (15.8\%), SafeWork SA (15.1\%), the internet (8.1\%), and newspapers $(5.5 \%)$. Some $10.3 \%$ of respondents stated that they could not access any information about heat stress prevention.

Respondents were asked if they worked at their own pace during very hot weather and the majority (70.4\%) answered in the affirmative. As to the reasons why the remaining $29.6 \%$ did not work at their own pace (Question 23, Additional file 1), more than two-thirds (68.0\%) attributed this to the pressure from work demands, followed by the pressure from supervisors (46.1\%), and peer pressure $(24.3 \%)$. In addition, $11.2 \%$ replied that there was no need to slow down their work rate during hot weather because "Enough has been done to cool the workplace".

\section{Current heat prevention measures}

As shown in Fig. 2, the provision of cool drinking water $(69.8 \%)$ was the most common prevention measure adopted in the workplace against heat exposure in South Australia, followed by wearing broad brimmed hats (39.0\%), rescheduling work time (33.8\%), central cooling and air conditioning (33.6\%), electric fans (33.6\%), 


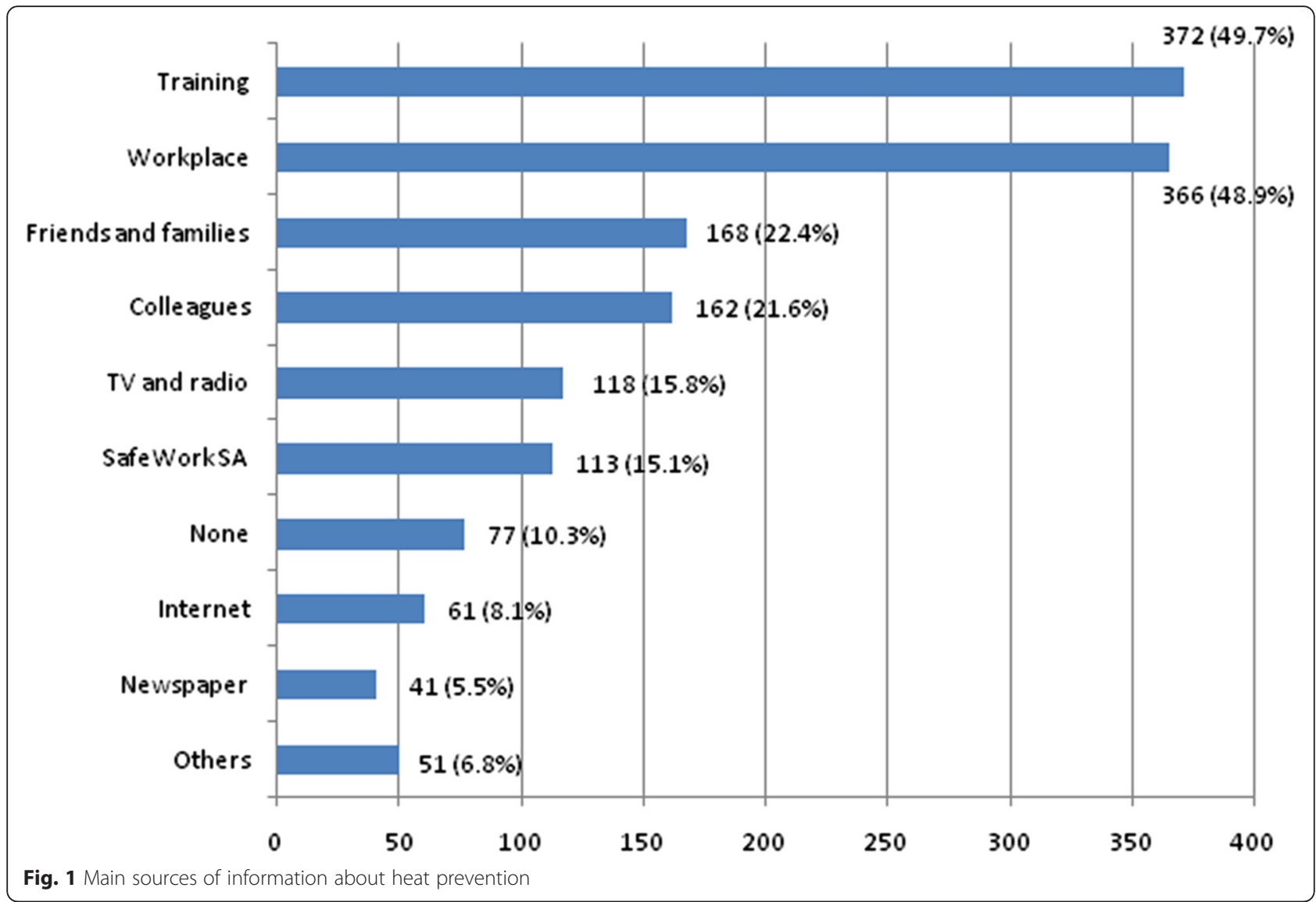

and shady rest area (33.1\%). Only $19.6 \%$ answered "stopping work" as a prevention measure when the temperature was extremely hot (e.g., $>40{ }^{\circ} \mathrm{C}$ ).

When asked if there were guidelines for heat stress prevention during extremely hot weather, $50.9 \%$ of respondents answered "Yes". Whilst $43.4 \%$ had attended a heat- related training course, $60.0 \%$ participants had received instructions on first aid procedures for serious heat illnesses.

\section{Discussion}

It is important to understand workers' perceptions on workplace extreme heat exposure in a warming climate,

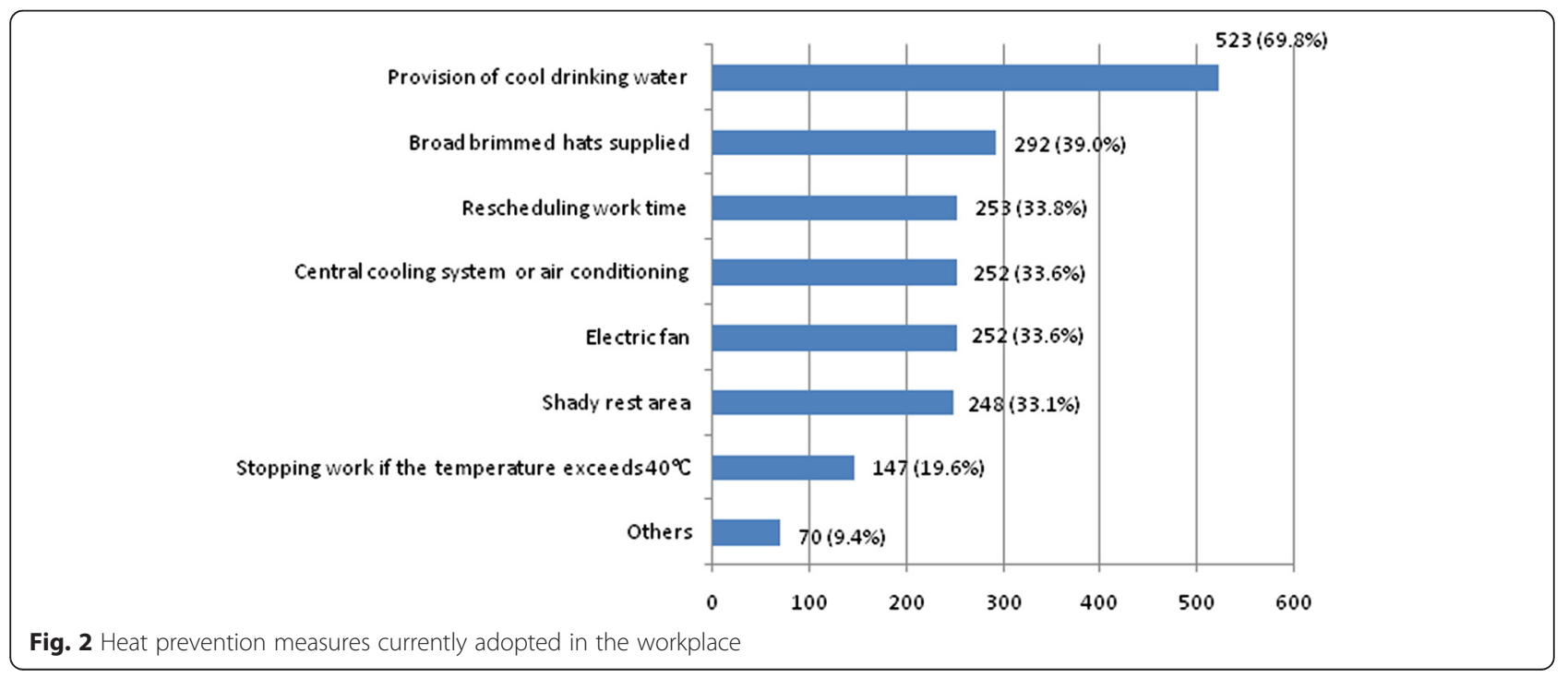


as this information may provide evidence for updating heat prevention strategies to reduce the impact of climate change on workers' health and safety. However, most of the currently available information is from qualitative studies in middle-low income countries [2426]. Limited evidence suggests that workers' perceptions regarding likely increasing extreme heat exposure due to climate change in high income countries are not optimistic [11]. This survey is a modest step to investigate workers' heat risk perceptions in a developed country, using a quantitative approach.

\section{Workplace heat exposure concern in Australia}

This study revealed that about half of the respondents were moderately to very much concerned about their occupational health and safety when working during very hot weather. The percentage $(50 \%)$ concerned in those participants employed in the "agriculture, forestry and fishing" industry in this study was about 6 times higher than that $(8 \%)$ in a study of Californian hired farmworkers in the USA [27]. The relatively higher heat awareness among the Australian workplace may reflect cultural and demographic differences in study populations.

Our results suggest that young workers were less concerned about heat exposure than older workers. Moreover, middle aged workers supported having more heat-related training compared to young and older workers. There are many concerns about Australian young workers' attitudes towards occupational health and safety, of which heat stress is only one. According to the national "Motivations, Attitudes, Perceptions and Skills" survey, up to $28 \%$ of Australian young workers reported resenting dealing with workplace health and safety requirements, and $42 \%$ forgot about safety during working practice [28]. Evidence has shown that young workers are at high risk of heat-related illnesses and injuries when the temperature is below a certain threshold (e.g., $\left.37.9^{\circ} \mathrm{C}\right)[2,4,5]$. By contrast, older workers are more vulnerable to heat-related illnesses/injuries in certain outdoor industries during heatwaves [3]. Therefore, more educational programmes should be targeted to these two age groups. Further research is also needed to explore the paradoxical phenomena that young workers were less satisfied with heat prevention measures but expressed more negative attitudes towards heat prevention efforts, compared with older workers. Probably, it is because young workers were relatively more powerless than older workers in the workplace and held negative attitudes towards their management [21]. The compliance and implementation of heat prevention and adaptation policies would be undermined if the sentiments of unwillingness to cooperate existed among young workers [28].
Undertaking very physically demanding work and wearing PPE was also found to be associated with workers' concern about heat exposure. Both of these are very important factors determining human body heat balance, and can be indirectly reflected in the standard workplace heat stress management procedure [8]. The results also suggest that workers who had a previous heat-related illness and/or injury were more concerned about heat exposure.

\section{Heat-related training}

In this study, about $43 \%$ of respondents indicated that they have received heat-related training. The relatively higher proportion of workers receiving heat prevention training in this study maybe partly because of the lessons learnt from the past. Previously, less $\mathrm{OH} \& \mathrm{~S}$ training and education had been provided in Australian workplaces, and this was identified as one of the top three causes of occupational injuries and accidents in Australia [29]. Therefore, relevant work health and safety training has been incorporated into secondary, vocational and university level education since the mid-to late 1990s in Australia [29].

The results indicated that heat training was the workers' major source of information about heat stress prevention in Australia, and this is the case for occupational health and safety in general [29]. Evidence has suggested that the majority of outdoor physical workers in the USA [27, 30], India [26] and South Africa [24] had a good knowledge of the symptoms and severe outcomes of excessive heat exposure [24, 27, 30]. Although workers' average level of knowledge on heat stress wasn't investigated in this study, $16 \%$ of respondents said they only drank when they were thirsty. This indirectly reflects the necessity to reinforce messages about dehydration in the workplace. Relevant training and education should focus on young workers and those aged over 55 years, as these groups expressed less willingness to receive more heat training. Moreover, studies have shown they are at relatively higher risk of heat-related illness and injury [2-4]. More supportive attitudes towards heat-related training in the age group of 25-54 years may account for their greater concerns over heat exposure. The reasons why workers aged $\geq 55$ years did not show stronger willingness to support more heatrelated training whilst being more concerned about heat compared to young workers, may include that they may be more satisfied with current preventive measures in place or they may undertake more sedentary jobs with less heat exposure. In addition to training in the workplace, the role of mass media in popularizing heat stress prevention knowledge should be strengthened, as up to $10 \%$ of respondents in this study claimed to have had no sources of heat prevention information. 


\section{Individual behavioural response}

The majority of respondents expressed their willingness to adjust work habits to adapt to possible increasing hot weather, and this may be useful for future heat intervention measures. The results from this study suggested that previous heat illness experience was the only factor associated with the adjustment of work habits, indicating a need for improving heat risk awareness. Moreover, a good level of heat stress knowledge and awareness does not necessarily translate into individual behavioural change [30]. The knowledge-behaviour gaps may provide opportunities for additional heat prevention and education strategies.

In other studies, self-pacing (adjusting work rate to avoid physiological heat strain) has been used to explain why workers were not heat stressed when working in hot environments $[13,31]$. In the present study, up to $70 \%$ of respondents expressed that they worked at their own pace during very hot weather. For others, pressure from work demand and supervisors was the major reason that workers did not slow down their work rate. Most recently, Lao et al. interviewed 32 male council workers in South Australia and found they had a high level of heat resilience through personal adaptive behaviours [21]. Nevertheless, profit-oriented production and performance targets have been shown to be a common reason overshadowing or marginalizing heat stress prevention [11, 21]. Employee-based behavioural change is not enough to reduce heat-related illness and injury, as employees may be powerless in an occupational health and safety management system [11, 21, 32]. Relevant heat prevention campaigns and legislations should target employers as heat stress not only impacts workers' health and safety but also may compromise productivity $[1,6,11,12,33]$. However, to date few studies have investigated how employers in industry perceive the risk of heat exposure, although results of our recently published paper showed that the majority of occupational hygienists and specialists in Australia were concerned about workplace extreme heat exposure [34].

\section{Heat prevention measures}

In this study about one-quarter of respondents claimed to have witnessed heat-related illnesses or injuries during extremely hot weather. This may indirectly reflect the high incidence of occupational heat illnesses despite only 306 heat-related compensation claims being identified in South Australia during the period of 2001-2010 with an incidence rate of 4.5 per 100000 employees [35]. This figure may be underestimated due to underreporting and misclassification [13]. Fortunately, all heat-related illnesses, injuries and deaths are largely preventable. Heat prevention strategies mainly include regulations, administrative controls, and engineering modifications.
Currently, there are systematic technical guidelines and manuals in place for heat stress monitoring, risk assessment, control and prevention, such as ISO (International Organization for Standardization) heat indices [36], ACGIH (American Conference of Governmental Industrial Hygienists) [37] and NIOSH (US National Institute for Occupational Safety and Health) heat standards [38]. Based on above heat standards, the Australian Institute of Occupational Hygienists (AIOH) has developed a heat stress management guideline for use in the Australian environment [8]. However, it should be realized that these guidelines and technical manuals do not have a legal force.

It has been proven that without proper enforcement heat regulations are likely to pose little restrictions to non-compliant employers [39, 40]. In 2010, California became the first State in the USA to enact a stringent heat-specific law to protect workers from heat exposure [41]. Two years later however, inspectors found that more than half of the employers they audited did not comply with the heat standard [42]. Heat prevention measures seem straightforward, common-sense, and simple (e.g., drinking water frequently, wearing light coloured and permeable clothes, taking breaks in the shade, and responding to early symptoms). However, a variety of factors at multiple levels in the workplace may constrain such implementation, such as production quotas, worries of being considered 'soft', and workers' fears of losing their job $[11,21,39]$. Currently, there is no federal occupational standard specifically addressing heat illness and injury prevention in Australia [12, 35], which may make the implementation of heat prevention measures problematic.

Maintaining hydration is very important for heat prevention. In this study, approximately $30 \%$ of respondents replied that cool drinking water was not available in the workplace. Moreover, about $16 \%$ of respondents only drank when thirsty. Thirst cannot be relied upon as a guide for the need for water, as $1 \%$ of the total body weight in water is already lost when an individual senses thirst [43]. According to the national Model Code of Practice (managing the work environment and facilities) [44], "an adequate supply of clean drinking water must be provided free of charge for workers at all times." However, its implementation and effectiveness are questionable, as evidence has shown that a poor hydration status has been observed among workers employed in a range of industries in Australia [45, 46].

Our results showed that only $20 \%$ of respondents selected "stopping work" as a heat prevention measure when the temperature was extremely hot. However, according to the heat stress management policy of the Construction, Forestry, Mining and Energy Union (CFMEU) South Australia Branch, "if temperature is over $37{ }^{\circ} \mathrm{C}$ all work ceases unless working in an air 
conditioned area" [47]. In this study, the majority (67\%) of participants were recruited from agriculture, forestry, mining, construction, and "electricity, gas and water" industries, and about half worked outdoors. Therefore, this raises concerns regarding the compliance of heat policies.

In this study, about $64 \%$ of respondents thought there was a need for more heat-related regulations. Meanwhile, about half the respondents were not satisfied with current prevention measures, indicating the necessity and urgency of the development of heat policies, especially for young workers with low education levels and undertaking physically demanding work outdoors. Most recently, Jia and Rowlinson et al. [48] formulated a comprehensive socio-ergonomic framework for identifying heat risk factors in the construction industry, and suggested a set of localized, simplified, action-triggering and threshold-based guidelines for the development of current heat management system [49]. This may provide useful inspirations for developing more effective and practical heat stress management for the construction industry and other at-risk industries. Therefore, in addition to strengthening the implementation of current existing regulations during extremely hot days, more efforts are needed to develop local industry specific heat stress policies [49].

\section{Limitations}

There are several limitations in this study. First, the vast majority (96\%) of respondents were males. Caution should be used when generalizing the results to female workers. Moreover, as the participation of the survey was completely voluntary, those with previous heat illness and injury experience may have been more likely to participate in the survey, which may generate potential selection bias and therefore may overestimate workers' heat concerns. Second, the level of workers' heat-related knowledge was not specifically measured in this study. However, published papers have consistently found that most workers in both developing and developed countries have a good knowledge of heat illnesses [24, 27, 30, 50]. Third, the relatively low response rate (50.9\%) in this study may generate potential non-respondent bias. Although there is not necessarily a relationship between response rates and bias, non-response bias may occur if respondents significantly differ from non-respondents [51]. Fourth, $68.2 \%$ of participants were TAFE trainees rather than established workers, although the majority worked as apprentices on a part-time basis. Lastly, the survey was not conducted during the hottest part of the year, posing the opportunity for recall bias.

\section{Conclusion}

This study provides important insights and baseline information regarding workers' perceptions and attitudes towards workplace heat exposure. Workers in South
Australia were moderately concerned about heat exposure. Further heat educational programmes and training should focus on those undertaking physically demanding work outdoors, in particular young workers and those over 55 years with low education level. The high proportion of respondents unsatisfied with current heat prevention measures and supporting more heat-related regulations indicates the necessity to refine current workplace heat prevention policies in a warming climate. In addition, there is a need to develop local specific and clear enforceable heat regulations.

\section{Additional files}

Additional file 1: Survey questionnaire: extreme heat exposure and worker health \& safety. (PDF 256 kb)

Additional file 2: Information sheet for participants. (DOC 130 kb)

\section{Abbreviations}

CFMEU, Construction, Forestry, Mining and Energy Union; IPCC, intergovernmental panel on climate change; OH\&S, occupational health and safety; OSHA, occupational safety and health administration; PPE, personal protective equipment; SWSA, SafeWork South Australia; TAFE, technical and further education

\section{Acknowledgements}

The authors would like to thank SafeWork SA and TAFE SA for providing assistance in the distribution of questionnaires. All survey participants and employers getting involved in this study are greatly appreciated for their contributions. We also thank the reviewers for their valuable comments and suggestions.

\section{Funding}

This manuscript is part of JX's PhD Thesis. There is no funding for this study.

\section{Availability of data and materials}

Data is not available for online access. Only members of the research team have access to the data, according to the ethics approval $(\mathrm{H}-200-2011)$ and information sheet (Additional file 2).

\section{Authors' contributions}

JX designed and implemented the study, interpreted the results and drafted the manuscript. PB, DP, and AH designed and supervised the study, assisted with questionnaire design, statistical analyses and interpretation of results, reviewed and revised the manuscript. All authors read and approved the final version of the manuscript.

\section{Competing interests}

The authors declare that they have no competing interests.

\section{Consent for publication}

This manuscript does not contain any individual person's data. All participants were informed of the publication of research findings through information sheets. Permission to publish the results from this study was granted by the Human Research Ethics Committee at the University of Adelaide.

\section{Ethics approval and consent to participate}

Ethics approval was obtained from the Human Research Ethics Committee at the University of Adelaide (H-200-2011). Information sheets were provided to all participants for understanding the research objectives and procedure. Participation was voluntary and strictly confidential. Participants were allowed to withdraw from the study at any time or decline to answer any question. Oral consent was obtained from each participant. 


\section{Author details}

'School of Public Health, The University of Adelaide, Adelaide 5005, SA, Australia. ${ }^{2}$ Department of Emergency Response and Preparedness, Fujian Provincial Center for Disease Control and Prevention, Fuzhou 350001, China

Received: 8 January 2016 Accepted: 15 June 2016

Published online: 11 July 2016

\section{References}

1. Schulte PA, Chun H. Climate change and occupational safety and health: Establishing a preliminary framework. J Occup Environ Hyg. 2009;6(9):542-54.

2. Xiang J, Bi P, Pisaniello D, Hansen A, Sullivan T. Association between high temperature and work-related injuries in Adelaide, South Australia, 2001-2010. Occup Environ Med. 2014;71(4):246-52.

3. Xiang J, Bi P, Pisaniello D, Hansen A. The impact of heatwaves on workers health and safety in Adelaide South Australia. Environ Res. 2014;133C:90-5.

4. Fogleman M, Fakhrzadeh $L$, Bernard TE. The relationship between outdoor thermal conditions and acute injury in an aluminum smelter. Int J Ind Ergon. 2005;35(1):47-55

5. Morabito M, Cecchi L, Crisci A, Modesti PA, Orlandini S. Relationship between work-related accidents and hot weather conditions in Tuscany (central Italy). Ind Health. 2006;44(3):458-64.

6. Kjellstrom T, Kovats RS, Lloyd SJ, Holt T, Tol RS. The direct impact of climate change on regional labor productivity. Arch Environ Occup Health. 2009; 64(4):217-27.

7. Kjellstrom T, Lemke B, Otto M. Mapping occupational heat exposure and effects in South-East Asia: ongoing time trends 1980-2011 and future estimates to 2050. Ind Health. 2013;51(1):56-67.

8. Corleto RD, Firth I, Mate J. A guide to managing heat stress: developed for use in the Australian environment. 2nd ed. Tullamarine Victoria: The Australian Institute of Occupational Hygienists Inc (AIOH); 2013.

9. Commonwealth Scientific and Industrial Research Organization (CSIRO) and Australian Bureau of Meteorology (BOM). State of the Climate. 2014. http:// www.bom.gov.au/state-of-the-climate/. Accessed 2 July 2015.

10. Gubernot DM, Anderson GB, Hunting KL. The epidemiology of occupational heat exposure in the United States: a review of the literature and assessment of research needs in a changing climate. Int J Biometeorol. 2013:58(8):1779-88.

11. Singh S, Hanna EG, Kjellstrom T. Working in Australia's heat: Health promotion concerns for health and productivity. Health Promot Int. 2013. doi:10.1093/heapro/dat027.

12. Hanna EG, Kjellstrom T, Bennett C, Dear K. Climate change and rising heat: Population health implications for working people in Australia. Asia Pac J Public Health. 2010;23(2):14-26.

13. Xiang J, Bi P, Pisaniello D, Hansen A. Health impacts of workplace heat exposure: An epidemiological review. Ind Health. 2014;52(2):91-101.

14. Hyatt OM, Lemke B, Kjellstrom T. Regional maps of occupational heat exposure: past, present, and potential future. Glob Health Action. 2010;3. doi: 10.3402/gha.v3i0.5715

15. Maloney SK, Forbes CF. What effect will a few degrees of climate change have on human heat balance? Implications for human activity. Int J Biometeorol. 2011;55(2):147-60.

16. Mckean B. Bricklayer dies in heat. 2010. http://www.thechronicle.com.au/ news/bricklayer-dies-in-heat/488357/. Accessed 10 Nov 2014.

17. Australian Department of Commerce. Death of Queensland worker prompts warning on heat stress. 2009. http://www.commerce.wa.gov.au/corporate/ media/statements/2009/December/Death_of_Queensland_worker_pro.html. Accessed 4 May 2014

18. Cutforth P. Workers death linked to heat exposure. 2013. http://www. whitecardaustralia.com.au/blog/workers-death-linked-to-heat-exposure/. Accessed 8 May 2014.

19. Commonwealth Scientific and Industrial Research Organization (CSIRO) and Australian Bureau of Meteorology (BOM). Climate change in Australia Technical Report 2007-Chapter 5: Regional climate change projections (temperature and precipitation). http://ccia2007.climatechangeinaustralia. gov.au/. Accessed 31 July 2014

20. The US Department of Labor. Campaign to prevent heat illness in outdoor workers. 2011. https://www.osha.gov/SLTC/heatillness/index.html. Accessed 23 Aug 2014.
21. Lao J, Hansen A, Nitschke M, Hanson-Easey S, Pisaniello D. Working smart: An exploration of council workers' experiences and perceptions of heat in Adelaide South Australia. Saf Sci. 2016;82:228-35. doi:10.1016/j.ssci.2015.09.026.

22. US Centers for Disease Control and Prevention. Preventing deaths, injuries and illnesses of young workers. Morb Mortal Wkly Rep. 2003;128:1-22. https://www.cdc.gov/niosh/docs/2003-128/pdfs/2003128.pdf.

23. Agho K, Stevens G, Taylor M, Barr M, Raphael B. Population risk perceptions of global warming in Australia. Environ Res. 2010;110(8):756-63.

24. Mathee A, Oba J, Rose A. Climate change impacts on working people (the HOTHAPS initiative): findings of the South African pilot study. Glob Health Action. 2010;3. doi:10.3402/gha.v3i0.5612.

25. Crowe J, van Wendel de Joode B, Wesseling C. A pilot field evaluation on heat stress in sugarcane workers in Costa Rica: What to do next? Glob Health Action. 2009;2. doi:10.3402/gha.v2i0.2062.

26. Balakrishnan K, Ramalingam A, Dasu V, Stephen JC, Sivaperumal MR, Kumarasamy D, et al. Case studies on heat stress related perceptions in different industrial sectors in southern India. Glob Health Action. 2010;3. doi: 10.3402/gha.v3i0.5635

27. Stoecklin-Marois M, Hennessy-Burt T, Mitchell D, Schenker M. Heat-related Illness Knowledge and Practices among California Hired Farm Workers in The MICASA Study. Ind Health. 2013:51(1):47-55.

28. Safe Work Australia. Motivations, Attitudes, Perceptions and Skills: Pathways to Safe Work. 2011. http://www.safeworkaustralia.gov.au/sites/SWA/about/ Publications/Documents/581/Motivation_Attitude_Perceptions_and_Skills_ Pathways_to_Safe_Work.pdf. Accessed 2 Mar 2014.

29. Safe Work Australia. Motivations, attitudes, perceptions and skills: What they said about work health and safety in 2010. 2011. http://www.safeworkaustralia. gov.au/sites/SWA/about/Publications/Documents/603/Motivations_attitudes_ perceptions_skills_what_they_said.pdf. Accessed 15 Mar 2015.

30. Lam M, Krenz J, Palmandez P, Negrete M, Perla M, Murphy-Robinson H, et al. Identification of barriers to the prevention and treatment of heat-related illness in Latino farmworkers using activity-oriented, participatory rural appraisal focus group methods. BMC Public Health. 2013;13:1004.

31. Miller V, Bates G, Schneider JD, Thomsen J. Self-pacing as a protective mechanism against the effects of heat stress. Ann Occup Hyg. 2011;55(5): $548-55$

32. Fleischer NL, Tiesman HM, Sumitani J, Mize T, Amarnath KK, Bayakly AR, et al. Public health impact of heat-related illness among migrant farmworkers. Am J Prev Med. 2013:44(3):199-206.

33. Kjellstrom T, Gabrysch S, Lemke B, Dear K. The 'Hothaps' programme for assessing climate change impacts on occupational health and productivity: An invitation to carry out field studies. Glob Health Action. 2009;2. doi:10. 3402/gha.v2i0.2082

34. Xiang J, Hansen A, Pisaniello D, Bi P. Perceptions of Workplace Heat Exposure and Controls among Occupational Hygienists and Relevant Specialists in Australia. PloS One. 2015;10. doi:10.1371/journal.pone.0135040.

35. Xiang J, Hansen A, Pisaniello D, Bi P. Extreme heat and occupational heat illnesses in South Australia, 2001-2010. Occup Environ Med. 2015;72(8):580-6.

36. Parsons K. Occupational health impacts of climate change: current and future ISO standards for the assessment of heat stress. Ind Health. 2013; 51(1):86-100.

37. The American Conference of Governmental Industrial Hygienists (ACGIH). ACGIH Heat Stress and Strain TLV. https://www.acgih.org/forms/store/ ProductFormPublic/heat-stress-and-strain-tlv-r-physical-agents-7th-editiondocumentation. Accessed 1 May 2012.

38. National Institute for Occupational Safety and Health (NIOSH). Criteria for a recommended standard: occupational exposure to heat and hot environments. 2016. https://www.cdc.gov/niosh/docs/2016-106/. Accessed 20 Mar 2016.

39. Riley K, Delp L, Cornelio D, Jacobs S. From agricultural fields to urban asphalt: the role of worker education to promote California's heat illness prevention standard. New Solut. 2012:22(3):297-323.

40. Nevarez J. OSHA compliance issues: OSHA heat stress fatality investigation of a Latino landscaping worker. J Occup Environ Hyg. 2013;10(6):D67-70.

41. California Code of Regulations. Heat illness prevention; Subchapter 7, Section 3395. http://www.dir.ca.gov/title8/3395.html. Accessed 12 Nov 2013.

42. Khokha S. Teen farmworker's heat death sparks outcry. 2008. http://www. npr.org/templates/story/story.php?storyld=91240378. Accessed 1 May 2014

43. US Department of Labor. OSHA Technical Manual (OTM) on heat stress Section III: Chapter 4. https://www.osha.gov/dts/osta/otm/otm_iii/otm_iii_ 4. html. Accessed 5 July 2013. 
44. Safe Work Australia. Model Code of Practice: Managing the work environment and facilities. 2011. http://www.safeworkaustralia.gov.au/ sites/swa/about/publications/pages/environment-facilities-cop. Accessed 5 Apr 2014.

45. Miller VS, Bates GP. Hydration of outdoor workers in north-west Australia. Journal of Occupational Health and Safety Australia and New Zealand. 2007; 23(1):79-88.

46. Brake DJ, Bates GP. Fluid losses and hydration status of industrial workers under thermal stress working extended shifts. Occup Environ Med. 2003; 60(2):90-6.

47. Australian Construction, Forestry, Mining and Energy Union (CFMEU). Inclement weather - Heat Policy. 2013. http://www.cfmeu.asn.au/branch/sa/ campaign/inclement-weather-heat-policy. Accessed 20 Apr 2014.

48. Jia YA, Rowlinson S, Ciccarelli M. Climatic and psychosocial risks of heat illness incidents on construction site. Appl Ergon. 2016;53(Pt A):25-35. doi: 10.1016/j.apergo.2015.08.008.

49. Rowlinson S, Jia YA. Application of the predicted heat strain model in development of localized, threshold-based heat stress management guidelines for the construction industry. Ann Occup Hyg. 2014;58(3):326-39.

50. Flocks J, Vi Thien Mac V, Runkle J, Tovar-Aguilar JA, Economos J, McCauley LA. Female farmworkers' perceptions of heat-related illness and pregnancy health. J Agromedicine. 2013;18(4):350-8.

51. Asch DA, Jedrziewski MK, Christakis NA. Response rates to mail surveys published in medical journals. J Clin Epidemiol. 1997;50(10):1129-36.

\section{Submit your next manuscript to BioMed Central} and we will help you at every step:

- We accept pre-submission inquiries

- Our selector tool helps you to find the most relevant journal

- We provide round the clock customer support

- Convenient online submission

- Thorough peer review

- Inclusion in PubMed and all major indexing services

- Maximum visibility for your research

Submit your manuscript at www.biomedcentral.com/submit 